\title{
First report of the molecular detection of human pathogen Rickettsia raoultii in ticks from the Republic of Korea
}

\author{
Misbah Tariq ${ }^{1 \dagger}$, Jun-Won Seo ${ }^{1}$, Da Young Kim', Merlin Jayalal Lawrence Panchali ${ }^{2}$, Na Ra Yun ${ }^{1}$, You Mi Lee \\ Choon-Mee $\mathrm{Kim}^{2+}$ and Dong-Min Kim ${ }^{1 *}$ (1)
}

\begin{abstract}
Background: Rickettsial diseases associated with the spotted fever group constitute a growing number of newly identified Rickettsia pathogens and their tick vectors in various parts of the world. At least 15 distinct tick species belonging to six genera have shown the presence of Rickettsia raoultii. Herein, we report the detection of R. raoultii in ticks from the Republic of Korea (ROK).

Methods: Thirty-five ticks were collected from 29 patients with tick bites in Gwangju Metropolitan City, Jeollanam Province, ROK. The ticks were identified using molecular, morphological, and taxonomic characteristics. All samples were screened for presence of Rickettsia species using nested polymerase chain reactions of their outer membrane protein (ompA) and citrate synthase ( $g / t A)$ genes. The amplified products were sequenced for subsequent phylogenetic analyses.
\end{abstract}

Results: Sequencing data showed the DNA sequences of R. raoultii in three Haemaphysalis longicornis ticks. All three tick samples were $99.4-100 \%$ similar to previously reported partial sequences of ompA of R. raoultii strains CP019435 and MF002523, which formed a single clade with the reference strains.

Conclusions: We provide the first description and molecular identification of $R$. raoultii detected in $\mathrm{H}$. longicornis ticks in the ROK. This observation extends the geographical distribution of $R$. raoultii. Screening of human samples for this pathogen will provide information about the prevalence of rickettsial infections in this region.

Keywords: Rickettsia raoultii, Ticks, Haemaphysalis longicornis, Spotted fever group

\section{Background}

Tick-borne diseases are a growing medical concern worldwide. Ticks are considered the main reservoirs and vectors of Rickettsia, an obligate intracellular bacteria, responsible for the transmission of rickettsial diseases to humans. The rickettsioses represent some of the oldest and most recently recognized infectious

\footnotetext{
${ }^{*}$ Correspondence: drongkim@chosun.ac.kr

${ }^{+}$Misbah Tariq and Choon-Mee Kim contributed equally to this work

${ }^{1}$ Department of Internal Medicine, College of Medicine, Chosun University, Gwangju, Republic of Korea

Full list of author information is available at the end of the article
}

diseases [1]. The causative agents belong to the genus Rickettsia and are presently classified into four groups: the spotted fever group (SFG), typhus group, Rickettsia bellii group, and Rickettsia canadensis group [2]. SFG rickettsioses constitute newly identified Rickettsia species around the world. In the past few decades, numerous species of tick-borne rickettsiae, previously thought to be non-pathogenic, were recognized as human pathogens [2].

In 1999, three novel rickettsial genotypes, RpA4, DnS14, and DnS28, were observed in ticks from Russia [3]. Using genotypic and phenotypic analyses, these 
bacteria were recognized as novel species of SFG rickettsiae, and in 2008, the species was designated Rickettsia raoultii [4]. The major clinical manifestations of $R$. raoultii infections include scalp eschar and neck lymphadenopathy. Initially, these were termed Dermacentor-borne necrosis erythema and lymphadenopathy or tick-borne lymphadenopathy [2]. $R$. raoultii has been identified in many Asian and European countries [5-8]. In 1999, Dermacentor nuttalli and Rhipicephalus pumilio ticks collected in the southern parts of the former Soviet Union were shown to harbor these bacteria [3]; thereafter, other species of Dermacentor ticks (i.e., D. reticulatus, D. marginatus, $D$. silvarum, and $D$. niveus) from various parts of the former Soviet Union, as well as from France, Spain, and Germany, were also shown to carry these bacteria [5, 9]. Subsequently, $R$. raoultii was detected in other hard ticks too, such as Haemaphysalis, Rhipicephalus, Hyalomma, and Ambylomma, which are observed predominantly in Europe and Asia [10]. The aim of this study was to determine the presence of $R$. raoultii in ticks and to assess the circulation of this pathogen in tick populations in the Republic of Korea (ROK). We found $R$. raoultii in Haemaphysalis longicornis ticks. To the best of our knowledge, this is the first report providing molecular evidence of $R$. raoultii in ticks from the ROK.

\section{Methods}

\section{Tick sampling and classification}

In 2018, 35 ticks were collected from 29 patients with a history of tick bites in Gwangju Metropolitan City, Jeollanam Province, ROK. Ticks were identified on the basis of their molecular, morphological, and standard taxonomic characteristics. Briefly, the ticks were first decontaminated using $70 \%$ ethanol, rinsed twice using sterile phosphate-buffered saline (PBS), and dried on sterile filter paper. Each sample was then placed in a hard-tissue-grinding MK28 tube (Bertin Technology, Rockville, MD, USA) containing $800 \mu \mathrm{l}$ $\mathrm{PBS}$ and $1 \times \mathrm{PC} / \mathrm{SM}$ (i.e., penicillin and streptomycin). Subsequently, ticks were ground using a FastPrep-24 Classic instrument (MP Biomedicals, Solon, OH, USA) and were stored at $-80^{\circ} \mathrm{C}$ until DNA extraction.

\section{DNA extraction}

Total genomic DNA was extracted from $150 \mu \mathrm{l}$ of the tick homogenate and from $300 \mu \mathrm{l}$ of whole blood of the respective patients using a QIAamp Tissue \& Blood Mini Kit (Qiagen, Hilden, Germany) according to the manufacturer's instructions; the DNA was eluted in volumes of $50 \mu \mathrm{l}$ and $100 \mu \mathrm{l}$, respectively. The samples were stored at $-20{ }^{\circ} \mathrm{C}$ until polymerase chain reaction (PCR) amplification.

\section{PCR amplification}

For molecular identification, tick genomic DNA was subjected to PCR amplification of a fragment of the mitochondrial 16S rRNA gene [11]. To assess the presence of Rickettsia species in ticks and patients, genomic DNA samples were subjected to a nested PCR targeting the outer membrane protein $\mathrm{A}(\operatorname{omp} A)$ and citrate synthase $(g l t A)$ genes $[12,13]$. PCR primers and the respective product sizes are shown in Table 1 . The reactions were carried out in a total volume of $20 \mu \mathrm{l}$, comprising 16 $\mu \mathrm{l}$ distilled water, $1 \mu \mathrm{l}$ of each primer $(10 \mathrm{pmol} / \mu \mathrm{l})$, and $2 \mu \mathrm{l}$ genomic DNA template using AccuPower PCR PreMix (Bioneer, Daejeon, ROK). The PCR analysis was performed using an $\mathrm{AB}$ thermal cycler (Applied Biosystems, Foster City, CA, USA). A positive control with $R$. conorii DNA and a negative control with distilled water instead of template DNA were included in each set of PCR. The amplified products were analyzed by electrophoresis using a $1.2 \%$ agarose gel containing ethidium bromide and then visualized by using an ultra-violet transilluminator system (FAS-III, Toyobo, Osaka, Japan). A 100-bp ladder (Bioneer Corp, Korea) was used as a molecular weight marker.

\section{Phylogenetic analysis}

The PCR products were purified using a QIAquick PCR purification kit (Qiagen) and were sequenced in both directions by a commercial service provider (Solgent Inc, Daejeon, Korea). To analyze the percentage of similarity, the resulting sequences were correlated for identity with sequences from GenBank using the Basic Local Alignment Search Tool (BLAST) program. The neighbor-joining method was employed to produce a phylogenetic tree with the ClustalW algorithm of the MegAlign program (DNASTAR, Madison, WI, USA). Bootstrap analysis was performed to test the stability of the phylogenetic tree acquired through the neighbor-joining method.

\section{Results}

Molecular, morphological, and taxonomic characteristics revealed that 4 of the 35 ticks were Ixodes nipponensis, 14 were Amblyomma testudinarium, and 17 were $H$. longicornis (Table 2). PCR tests to amplify the ompA and gltA gene fragments for identification of SFG rickettsial disease agents were conducted on all 35 ticks. Sequencing data of the amplified ompA gene fragment revealed one distinct Rickettsia species in three H. longicornis ticks, which was identified as $R$. raoultii. Morphological and taxonomic characteristic showed that these ticks were adult females. The PCR targeting the gltA gene did not reveal any distinct Rickettsia species. 
Table 1 Oligonucleotide primers that were used to perform PCR in this study to detect the molecular targets in Rickettsia species

\begin{tabular}{llll}
\hline Target & Primer & Nucleotide sequence (5'-3') & Fragment length \\
\hline ompA & RR190.70F & ATGGCGAATATTTCTCCAAAAA & 634 bp (first) \\
& RR190.701R & GTTCCGTTAATGGCAGCATCT & \\
& RR190.70F & ATGGCGAATATTTCTCCAAAAA & 535 bp (nested) \\
& RR190.602R & AGTGCAGCATTCGCTCCCCCT & \\
gltA & GLTA1F & GACGGTGATAAAGGAATCTTG & 1022 bp (first) \\
& GLTA1R & CATTTCTTTCCATTGTGCCATC & \\
& GLTA2F & CTACGAACTTACCGCTATTAG & 446 bp (nested) \\
& GLTA2R & GACCAAAACCCATTAACCTAAAC & \\
\hline
\end{tabular}

Even though the three $R$. raoultii-positive ticks were collected from patients, blood samples from the respective three patients did not show $R$. raoultii infection, as assessed by PCR, nor did the patients show any symptoms suggesting infection by this pathogen. $R$. raoultii ompA sequences of all three tick samples were $99.4 \%-100 \%$ homologous to previously reported partial sequences of ompA from $R$. raoultii IM-16 strains isolated from CP019435 and MF002523. Using phylogenetic analyses, a neighbor-joining tree of Rickettsia species indicated that isolates of the current study belonged to a single clade with $R$. raoultii reference strains (Fig. 1). The bootstrap analyses statistically supported the main clustered sequence.

\section{Discussion}

Currently, 14 distinct tick species from 6 genera including Dermacentor (D. nuttalli, D. reticulatus, D. silvarum, and D. marginatus), Ambylomma (A. helvolum), Haemaphysalis (H. concinna, H. japonica, $H$. erinacei, and H. longicornis), Hyalomma (Hy. asiaticum and Hy. lusitanicum), Ixodes (I. persulcatus and I. ricinus), and Rhipicephalus (Rh. pumilio and $R h$. turanicus) have shown the presence of $R$. raoultii $[14,15]$. This bacterium was also found in Melophagus ovinus, a louse fly or sheep ked [16]. Dermacentor ticks are considered the main hosts and natural reservoirs of $R$. raoultii all over Europe and in a few countries in Asia, including China and Mongolia [17].

In the ROK, the first evidence of SFG rickettsiae in ticks was reported in 2003, followed by the first case of SFG rickettsiosis (Japanese spotted fever) in a patient in $2005[18,19]$. Over a period of 16 years, various species of SFG rickettsiae have been identified in ticks $(R$. japonica, $R$. monacensis, and $R$. rickettsii) and humans $(R$. japonica and $R$. monacensis) in the ROK [18-24]. Thus far, $R$. raoultii had not been identified in this region, and the present study reports its detection for the first time. Previously, only one study from China indicated the presence of $R$. raoultii in H. longicornis ticks [15]. Different strains of $R$. raoultii including Marne, 8/9 Karaganda, Khabarovask ${ }^{\mathrm{T}}$, Shayman, and IM 16 have been documented in Europe, Russia, and China [5, 10]. The phylogenetic tree produced in the current study showed that the positive samples formed a distinct clade at a high (100) bootstrap value with $R$. raoultii strain IM 16 from China. DNA sequences of $R$. japonica, $R$. monacensis, and $R$. rickettsii have been identified in $H$. longicornis from ROK $[10,18]$. According to a recent study, $H$. longicornis is the most prevalent tick species in the ROK (88.9\%), showing a nationwide distribution [24]. Despite its identification in multiple tick species, reports on human infections with $R$. raoultii are still lacking. Infections of patients with $R$. raoultii have been reported in Europe, the Far East of Russia, and a few cases in China [25]. Another study from China [17] identified $R$. raoultii DNA in clinical samples, apart from positive serological reports in patients from other countries. Based on these findings, $R$. raoultii is considered a human pathogen [26]. The observations of the current study indicate the presence of $R$. raoultii in ticks in the ROK, which warrants further research.

\section{Conclusions}

Our results provide evidence for the first instance to our knowledge of the identification of $R$. raoultii in ticks from the ROK. Detection of Rickettsia species in H. longicornis ticks suggests that these ticks may be a vector of

Table 2 Tick species observed in this study based on the stages of development and sex, as per their collection from patients with tick bites

\begin{tabular}{|c|c|c|c|}
\hline Tick species & $\begin{array}{l}\text { Haemaphysalis } \\
\text { longicornis }\end{array}$ & $\begin{array}{l}\text { Amblyomma } \\
\text { testudinarium }\end{array}$ & $\begin{array}{l}\text { Ixodes } \\
\text { nipponensis }\end{array}$ \\
\hline \multicolumn{4}{|c|}{ Developmental stage } \\
\hline Adult female & 13 & - & 4 \\
\hline Adult male & - & 5 & - \\
\hline Nymph & 1 & 9 & - \\
\hline Larva & 3 & - & - \\
\hline \multirow[t]{2}{*}{ Total no. } & 17 & 14 & 4 \\
\hline & 35 & & \\
\hline
\end{tabular}




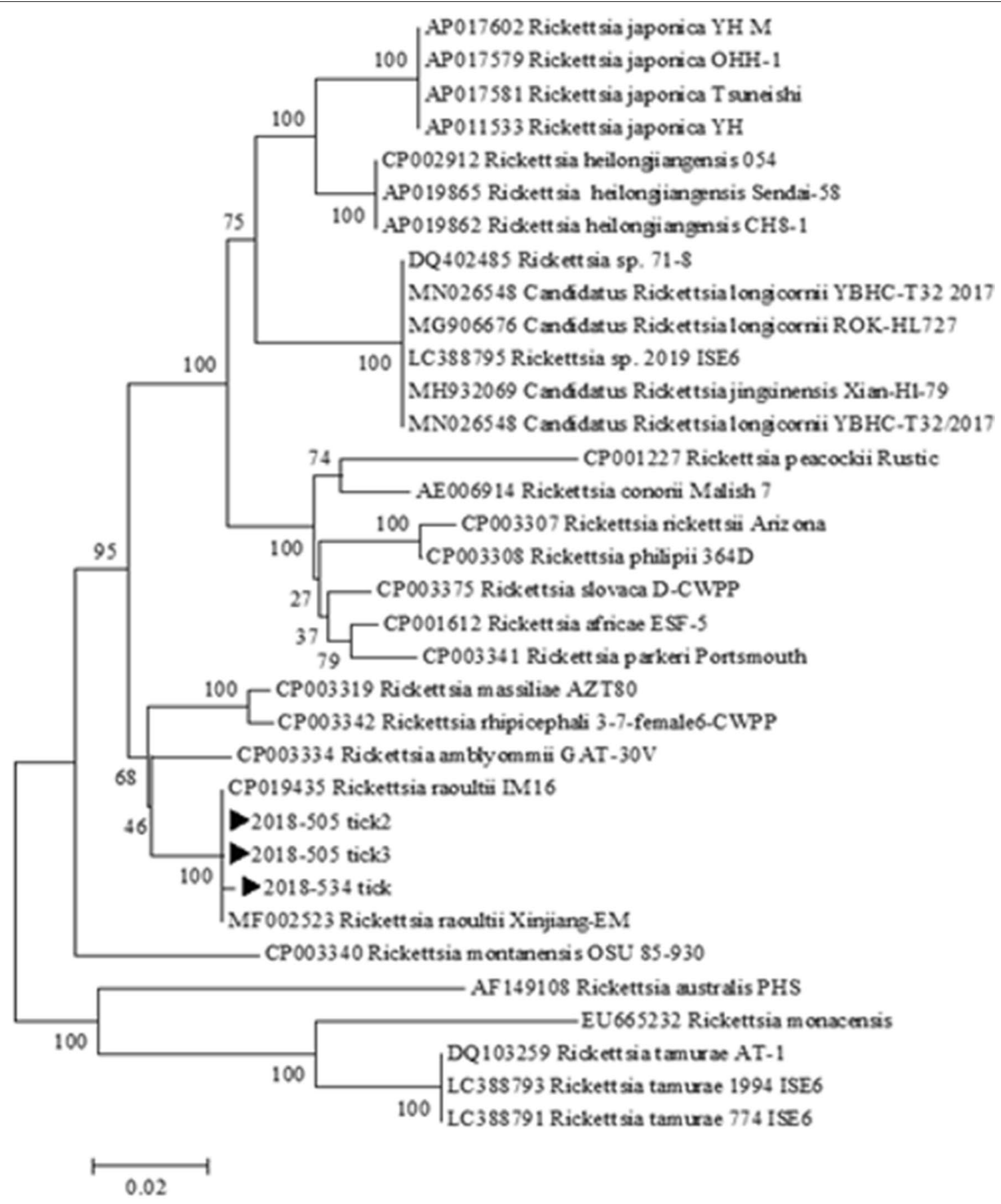

Fig. 1 Phylogenetic analysis of Rickettsia based on a partial (487-bp) ompA gene. Concatenated sequences are Rickettsia raoultii from Haemaphysalis longicornis ticks acquired in this study $(-)$ and sequences of Rickettsia downloaded from the GenBank database. The phylogenetic tree was produced using a neighbor-joining algorithm (NJ-1000 bootstrap trials)

this pathogen in the ROK. This observation broadens our knowledge of the geographical distribution of $R$. raoultii. Even though no human clinical infection was observed, the high pathogenicity of this bacterium is a major concern for public health in this region. Further extensive research in a broader range of ticks and surveillance programs in this regard are therefore required. 


\section{Abbreviations}

SFG: Spotted fever group; ROK: Republic of Korea; ompA: Outer membrane protein A; PBS: Phosphate-buffered saline; PCR: Polymerase chain reaction.

\section{Acknowledgements}

Not applicable.

\section{Authors' contributions}

D-MK designed and coordinated the study and contributed to drafting and reviewing the manuscript. MT and J-WS collected the data, wrote the manuscript, and revised the draft. MJLP performed the molecular analyses. YML constructed the phylogenetic tree. C-MK carried out the molecular analysis, wrote the manuscript, and revised the draft. NRY and D-YK helped drafting the manuscript. All authors read and approved the final manuscript.

\section{Funding}

This study was supported by research fund from Chosun University Hospital 2019.

\section{Availability of data and materials}

The datasets analyzed during the current study are available in the GenBankdatabase with the previously reported accession numbers; CP019435 and MF002523.

\section{Declarations}

\section{Ethics approval and consent to participate}

Not applicable.

\section{Consent for publication}

Not applicable.

\section{Competing interests}

The authors have no competing interests to declare.

\section{Author details}

${ }^{1}$ Department of Internal Medicine, College of Medicine, Chosun University, Gwangju, Republic of Korea. ${ }^{2}$ Department of Premedical Science, College of Medicine, Chosun University, Gwangju, Republic of Korea.

Received: 7 October 2020 Accepted: 20 March 2021

Published online: 07 April 2021

\section{References}

1. Raoult D, Roux V. Rickettsioses as paradigms of new or emerging infectious diseases. Clin Microbiol Rev. 1997;10(4):694-719.

2. Parola P, Paddock CD, Socolovschi C, Labruna MB, Mediannikov O, Kernif T, et al. Update on tick-borne rickettsioses around the world: a geographic approach. Clin Microbiol Rev. 2013;26(4):657-702.

3. Rydkina E, Roux V, Fetisova N, Rudakov N, Gafarova M, Tarasevich I, et al. New Rickettsiae in ticks collected in territories of the former Soviet Union. Emerg Infect Dis. 1999;5(6):811.

4. Mediannikov O, Matsumoto K, Samoylenko I, Drancourt M, Roux V, Rydkina E, et al. Rickettsia raoultii sp. nov., a spotted fever group rickettsia associated with Dermacentor ticks in Europe and Russia. Int J Syst Evol Microbiol. 2008;58(7):1635-9.

5. Nijhof AM, Bodaan C, Postigo M, Nieuwenhuijs H, Opsteegh M, Franssen $L$, et al. Ticks and associated pathogens collected from domestic animals in the Netherlands. Vector-Borne Zoonotic Dis. 2007;7(4):585-96.

6. Marquez F, Rojas A, Ibarra V, Cantero A, Rojas J, Oteo J, et al. Prevalence data of Rickettsia slovaca and other SFG rickettsiae species in Dermacentor marginatus in the southeastern Iberian peninsula. Ann NY Acad Sci. 2006;1078(1):328-30.

7. Wölfel R, Essbauer S, Dobler G. Diagnostics of tick-borne rickettsioses in Germany: a modern concept for a neglected disease. Int J Med Microbiol. 2008:298:368-74
8. Sumrandee C, Hirunkanokpun S, Doornbos K, Kitthawee S, Baimai V, Grubhoffer L, et al. Molecular detection of Rickettsia species in Amblyomma ticks collected from snakes in Thailand. Ticks Tick-borne Dis. 2014;5(6):632-40

9. Li H, Zhang P-H, Huang Y, Du J, Cui N, Yang Z-D, et al. Isolation and identification of Rickettsia raoultii in human cases: a surveillance study in 3 medical centers in China. Clin Infect Dis. 2018;66(7):1109-15.

10. Wen J, Jiao D, Wang J-H, Yao D-H, Liu Z-X, Zhao G, et al. Rickettsia raoultii, the predominant Rickettsia found in Dermacentor silvarum ticks in ChinaRussia border areas. Exp Appl Acarol. 2014;63(4):579-85.

11. Tian Z, Liu G, Xie J, Yin H, Luo J, Zhang L, et al. Discrimination between Haemaphysalis longicornis and $H$. qinghaiensis based on the partial $16 S$ rDNA and the second internal transcribed spacer (ITS-2). Exper Appl Acarol. 2011;54(2):165-72

12. Regnery RL, Spruill CL, Plikaytis B. Genotypic identification of rickettsiae and estimation of intraspecies sequence divergence for portions of two rickettsial genes. J Bacteriol. 1991;173(5):1576-89.

13. Jado I, Oteo JA, Aldámiz M, Gil H, Escudero R, Ibarra V, et al. Rickettsia monacensis and human disease, Spain. Emerg Infect Dis. 2007;13(9):1405.

14. Guo L-P, Mu L-M, Xu J, Jiang S-H, Wang A-D, Chen C-F, et al. Rickettsia raoultii in Haemaphysalis erinacei from marbled polecats China-Kazakhstan border. Parasit Vect. 2015;8(1):1-3.

15. Zhuang L, Du J, Cui X-M, Li H, Tang F, Zhang P-H, et al. Identification of tick-borne pathogen diversity by metagenomic analysis in Haemaphysalis longicornis from Xinyang China. Infect Dis Poverty. 2018;7(1):1-8.

16. Liu D, Wang Y-Z, Zhang H, Liu Z-Q, Wureli H-Z, Wang S-W, et al. First report of Rickettsia raoultii and $R$. slovaca in Melophagus ovinus, the sheep ked. Parasites Vect. 2016;9(1):1-6.

17. Jia N, Zheng Y-C, Ma L, Huo Q-B, Ni X-B, Jiang B-G, et al. Human infections with Rickettsia raoultii, China. Emerg Infect Dis. 2014;20(5):866.

18. Lee JH, Park HS, Jung KD, Jang WJ, Koh SE, Kang SS, et al. Identification of the spotted fever group rickettsiae detected from Haemaphysalis longicornis in Korea. Microbiol Immunol. 2003;47(4):301-4.

19. Choi Y-J, Jang W-J, Kim J-H, Ryu J-S, Lee S-H, Park K-H, et al. Spotted fever group and typhus group rickettsioses in humans, South Korea. Emerg Infect Dis. 2005;11(2):237

20. Jang WJ, Choi YJ, Kim JH, Jung KD, Ryu JS, Lee SH, et al. Seroepidemiology of spotted fever group and typhus group rickettsioses in humans South Korea. Microbiol Immunol. 2005;49(1):17-24

21. Choi YJ, Lee EM, Park JM, Lee KM, Han SH, Kim JK, et al. Molecular detection of various rickettsiae in mites (Acari: Trombiculidae) in southern Jeolla Province. Korea Microbiol Immunol. 2007:51(3):307-12.

22. Jiang J, An H, Lee JS, O'Guinn ML, Kim H-C, Chong S-T, et al. Molecular characterization of Haemaphysalis longicornis-borne rickettsiae, Republic of Korea and China. Ticks Tick-borne Dis. 2018;9(6):1606-13.

23. Noh Y, Lee YS, Kim H-C, Chong S-T, Klein TA, Jiang J, et al. Molecular detection of Rickettsia species in ticks collected from the southwestern provinces of the Republic of Korea. Parasit Vectors. 2017;10(1):1-10.

24. Im JH, Baek J, Durey A, Kwon HY, Chung M-H, Lee J-S. Current status of tick-borne diseases in South Korea. Vector-Borne Zoonotic Dis. 2019;19(4):225-33.

25. Dong Z, Yang Y, Wang Q, Xie S, Zhao S, Tan W, et al. A case with neurological abnormalities caused by Rickettsia raoultii in northwestern China. BMC Infect Dis. 2019;19(1):1-5.

26. Igolkina Y, Krasnova E, Rar V, Savelieva M, Epikhina T, Tikunov A, et al. Detection of causative agents of tick-borne rickettsioses in Western Siberia, Russia: identification of Rickettsia raoultii and Rickettsia sibirica DNA in clinical samples. Clin Microbiol Infect. 2018;24(2):199. e9-e12.

\section{Publisher's Note}

Springer Nature remains neutral with regard to jurisdictional claims in published maps and institutional affiliations. 